\title{
Ueber die Ernährung des Säugethierherzens.
}

\author{
Zweite Abhandlung. ${ }^{1}$
}

Von

Robert Tigerstedt.

(Aus dem physiologischen Laboratorium des Carolinischen medico-chirurgischen Instituts in Stockholm.)

1. In einer früheren Abhandlung habe ich gezeigt, dass man mittelst einer um die Vorhöfe gelegten, fest schliessenden Pincette beim Kaninchen die ganze Blutzufuhr nach den Kammern 5 Minuten lang abschneiden kann, ohne dass das Herz dadurch getödtet wird. Im Gegentheil fängt das Herz nach Entfernung der Pincette wieder an zu schlagen und der Kreislauf ist binnen Kurzem wieder ganz normal. ${ }^{2}$

Es bot ein gewisses Interesse dar, diesen Versuch am Hundeherzen zu wiederholen, weil dadurch die einander widerstreitenden Angaben über die nach Bindung der Kranzarterien beim Hundeherzen auftretenden Erscheinungen möglicherweise aufgeklärt werden könnten. ${ }^{3}$

Da, wie bekannt, das Hundeherz allen Eingriffen gegenüber viel empfindlicher als das Kaninchenherz ist, und es also zu erwarten war, dass die durch die Abklemmung hervorgerufenen Schädlichkeiten beim Hundeherzen schneller ihren deletären Einfluss ausüben sollten, fand ich es von vornherein angemessen, die abklemmende Pincette nicht allzu lange liegen za lassen. Um nämlich zu zeigen, dass der Herzstillstand u. s. w., welcher in den Versuchen von Cohnheim und Anderen bei Bindung einzelner Aeste der grossen Kranzarterie aufgetreten ist, in der

1 Der Redaction zugegangen den 4. December 1893.

- Tigerstedt, Dies Archiv, Bd. II, S. 394; 1890.

${ }^{3}$ In Bezug auf diese Untersuchungen vgl. Tigerstedt, Lehrbuch d. Physiologie des Kreislaufes, Leipzig 1893, S. 190-193, und die später erschienenen Abhandlungen von Porter, Journal of physiology, XV, S. 121-138; 1893; - Arch. f. d. ges. Physiol., LV, S. 366-371; 1893. 
That nicht von dem Blutmangel eines umschriebenen Bezirkes des Herzmuskels, sondern von Nebenverletzungen bedingt gewesen ist, schien es mir rollkommen genügend, die Abklemmung der Vorhöfe nur etwa ebenso lange wie bei den Versuchen Cohnheim's die Irranzarterienligatur dauern zu lassen. Ich kann daher keine Angaben über die maximale Zeit mittheilen, während welcher das Hundeherz in der von mir geübten Weise ohne Blutzufubr lebensfähig erhalten werden kann. Ich war gezwungen, meine Aufgabe in dieser Weise zu beschränken, weil Hunde geeigneter Grösse nur sehr spärlich zu meiner Verfügung standen.

2. In den beiden ersten jetzt mitzutheilenden Versuchen waren die Thiere nach Dastre und Morat durch subcutane Einspritzung ron Morphin und Atropin $\left(0.01\right.$ bezw. $0.001 \mathrm{~g}$ pro $\left.{ }^{\mathrm{kg}}\right)$ anästesirt und dann durch Curare bewegungslos gemacht.

Versuch I. 20. Februar 1892. Hund, 2100 grm. Morphin + Atropin + Curare in subcutaner Einspritzung.

\begin{tabular}{|c|c|c|c|c|c|}
\hline \multirow{2}{*}{ Nr. } & \multirow{2}{*}{$\begin{array}{l}\text { Laufende Zeit; } \\
\text { Sccunden }\end{array}$} & \multirow{2}{*}{$\begin{array}{l}\text { Puls in } \\
10 \text { Secunden }\end{array}$} & \multicolumn{3}{|c|}{ Blutdruck in der A. car.; $\mathrm{mm} \mathrm{Hg}$} \\
\hline & & & Max. & Min. & Mittel \\
\hline 1 & 0 & 23 & 35 & 31 & 33 \\
\hline 2 & 10 & 23 & 35 & 31 & 33 \\
\hline 3 & 20 & 24 & 35 & 31 & 33 \\
\hline 4 & 30 & 23 & 35 & 17 & 26 \\
\hline 5 & 40 & 24 & 29 & 17 & 23 \\
\hline 6 & 50 & 21 & 23 & 17 & 25 \\
\hline 7 & i0 & - & 20 & 5 & $12^{1} ;$ \\
\hline
\end{tabular}

Die Abklemmung der Vorhöfe findet in der 70. Secunde statt und dauert bis zu der 185. Secunde incl., also 115 Secunden lang. Dabei beträgt der Blutdruck $6-8^{\mathrm{mm}} \mathrm{Hg}$. Die Pincette wird bei 185 Secunden wieder fortgenommen.

\begin{tabular}{r|c|c|r|r|r}
\hline \hline Nr. & $\begin{array}{c}\text { Laufende Zeit; } \\
\text { Secunden }\end{array}$ & $\begin{array}{c}\text { Puls in } \\
\text { 10 Secunden }\end{array}$ & \multicolumn{3}{|c|}{$\begin{array}{c}\text { Blutdruck in der A. car.; mm Hg } \\
\text { Max. }\end{array}$} \\
\hline 8 & 186 & 16 & 11 & 3 & Min. \\
\hline 9 & 196 & 14 & 53 & 5 & 29 \\
10 & 206 & 15 & 45 & 25 & 35 \\
11 & 216 & 18 & 63 & 35 & 49 \\
12 & 226 & 24 & 97 & 59 & 78 \\
13 & 236 & 28 & 109 & 91 & 100 \\
14 & 246 & 29 & 119 & 103 & 111
\end{tabular}




\begin{tabular}{|c|c|c|c|c|c|}
\hline \multirow{2}{*}{ Nr. } & \multirow{2}{*}{$\begin{array}{c}\text { Laufende Zeit; } \\
\text { Secunden }\end{array}$} & \multirow{2}{*}{$\begin{array}{l}\text { Puls in } \\
10 \text { Secunden }\end{array}$} & \multicolumn{3}{|c|}{ Blutdruck in der A. car.; $\mathbf{m m ~} \mathrm{Hg}$} \\
\hline & & & Max. & ! Min. & Mittel \\
\hline 15 & 256 & 29 & 121 & 111 & 116 \\
\hline 16 & 266 & 29 & 117 & 101 & 109 \\
\hline 17 & 276 & 29 & 107 & 89 & 98 \\
\hline 18 & $2 \times 6 i$ & 29 & 95 & 83 & 89 \\
\hline 19 & 296 & 29 & 89 & 79 & 84 \\
\hline 20 & 306 & 29 & $8 \tilde{5}$ & 75 & 80 \\
\hline 21 & 316 & 29 & 81 & 69 & 75 \\
\hline 22 & 326 & 28 & 77 & 67 & 72 \\
\hline 23 & 336 & 28 & 83 & 65 & 74 \\
\hline 24 & 346 & 28 & 75 & 61 & 68 \\
\hline 25 & 356 & 27 & 71 & 59 & 65 \\
\hline 26 & 366 & 27 & 69 & 59 & 64 \\
\hline 27 & 376 & 26 & 67 & 57 & 62 \\
\hline 28 & 386 & 26 & 66 & 55 & $601 / 2$ \\
\hline 29 & 396 & 26 & 65 & 53 & 59 \\
\hline 30 & 741 & 24 & 49 & 43 & 46 \\
\hline 31 & 1161 & 24 & 39 & 33 & 36 \\
\hline 32 & 1171 & 23 & 39 & 33 & 36 \\
\hline 33 & 1181 & 24 & 41 & 33 & 37 \\
\hline 34 & 1751 & 23 & 35 & 31 & 33 \\
\hline 35 & 1761 & 23 & 35 & 31 & 33 \\
\hline 36 & 1771 & 22 & 37 & 27 & 32 \\
\hline
\end{tabular}

In der 1841. Sec. fängt eine neue Abklemmung der Vorhöfe an. Sie dauert 180 Sec. und dabei sinkt nach 50 Sec. der Blutdruck auf $5^{\mathrm{mm}} \mathrm{Hg}$, welchen Werth er bis zur Lösung der Klemme beibehält. Die Pulsfrequenz war, wegen Insufficienz der Aortaklappen, an der Blutdruckscurve ersichtlich und betrug während der einzelnen Perioden von 10 Sec.: $22-21-19-18-14-7-1-2-3$ $-2-1-2-2-1-2-1-1-2$. In der 2021. Sec. wird die Abklemmung aufgehoben.

\begin{tabular}{c|c|c|c|c|c}
\hline \hline Nr. & $\begin{array}{c}\text { Laufende Zeit; } \\
\text { Secunden }\end{array}$ & $\begin{array}{c}\text { Puls in } \\
\text { 10 Secunden }\end{array}$ & \multicolumn{3}{|c}{ Blutdruck in der A. car.; mm Hg } \\
& Max. & Min. & Mittel \\
\hline 37 & 2021 & 3 & - & - & 7 \\
38 & 2031 & 4 & 9 & 3 & 6 \\
39 & 2041 & 9 & 16 & 7 & $11^{1 / z}$ \\
40 & 2051 & 9 & 17 & 11 & 14 \\
41 & 2061 & 9 & 17 & 11 & 14 \\
42 & 2071 & 9 & 16 & 11 & $13^{1 / 4}$ \\
43 & 2081 & 9 & 16 & 11 & $13^{1 / 2}$ \\
44 & 2091 & 8 & 16 & 11 & $13^{1 / 2}$
\end{tabular}


Der Blutdruck erhebt sich nicht mehr. Die Herzschläge sind aber vollkommen normal und zeigen keine Spur eines Deliriums. Ein solches wird aber durch Kneten des Herzens hervorgerufen und das Thier stirbt.

Versuch II. 5. März 1892. Hund 10,000 grm. Morphin + Atropin + Curare in subcutaner Einspritzung.

\begin{tabular}{|c|c|c|c|c|c|c|}
\hline \multirow{2}{*}{ Nr. } & \multirow{2}{*}{$\begin{array}{l}\text { Laufende Zeit; } \\
\text { Secunden }\end{array}$} & \multirow{2}{*}{$\begin{array}{l}\text { Puls in } \\
10 \text { Secunden }\end{array}$} & \multicolumn{4}{|c|}{ Blutdruck in der A. car.; $\mathrm{mm} \mathrm{Hg}$} \\
\hline & & & Max. & Min. & 1 & Mittel \\
\hline$=$ & - & & & & & \\
\hline 1 & 0 & 28 & 92 & 85 & & $88^{1 / 2}$ \\
\hline 2 & 10 & 29 & 94 & 85 & & $89^{1} / 2$ \\
\hline 3 & 20 & 28 & 94 & 86 & & 90 \\
\hline 4 & 30 & 28 & 92 & 84 & & 88 \\
\hline 5 & 40 & 28 & 94 & 85 & & $89^{1} / 2$ \\
\hline 6 & 50 & 28 & 98 & 85 & & $911 / 2$ \\
\hline
\end{tabular}

Zwischen der 60. und 70. Sec. wird die Pincette angelegt. Die Abklemmung beginnt in der 72. Sec. und dauert bis zu der 206. Sec., also 134 Sec. lang. Dabei beträgt der Blutdruck $30 \mathrm{~mm} \mathrm{Hg}$. In der 206. Sec. wird die Pincette fortgenommen.

\begin{tabular}{|c|c|c|c|c|c|}
\hline \multirow{2}{*}{ Nr. } & \multirow{2}{*}{$\begin{array}{l}\text { Laufende Zeit; } \\
\text { Secunden }\end{array}$} & \multirow{2}{*}{$\begin{array}{l}\text { Puls in } \\
10 \text { Secunden }\end{array}$} & \multicolumn{3}{|c|}{ Blutdruck in der A. car.; $\mathrm{mm} \mathrm{Hg}$} \\
\hline & & & Max. & Min. & Mittel \\
\hline 7 & 206 & 17 & 140 & 30 & - \\
\hline 8 & 215 & 27 & 178 & 132 & 155 \\
\hline 9 & 225 & 30 & 182 & 164 & 173 \\
\hline 10 & 235 & 30 & 182 & 161 & $171^{1} / 2$ \\
\hline 11 & 245 & 30 & 176 & 154 & 165 \\
\hline 12 & 255 & 29 & 168 & 146 & 157 \\
\hline 13 & 265 & 29 & 158 & 136 & 147 \\
\hline 14 & 275 & 30 & 148 & 124 & 136 \\
\hline 15 & 285 & 29 & 138 & 110 & 124 \\
\hline 16 & 295 & 28 & 124 & 98 & 111 \\
\hline 17 & 305 & 28 & 110 & 90 & 100 \\
\hline 18 & 315 & 27 & 102 & 84 & 93 \\
\hline 19 & 425 & 27 & 88 & 78 & 83 \\
\hline 20 & 435 & 27 & 88 & 76 & 82 \\
\hline 21 & 810 & 29 & 92 & 82 & 87 \\
\hline 22 & 820 & 29 & 92 & 84 & 88 \\
\hline 23 & 830 & 30 & 92 & 82 & 87 \\
\hline
\end{tabular}




\begin{tabular}{|c|c|c|c|c|c|c|}
\hline \multirow{2}{*}{$\mathrm{Nr}$. } & \multirow{2}{*}{$\begin{array}{l}\text { Laufende Zeit; } \\
\text { Secunden }\end{array}$} & \multirow{2}{*}{$\begin{array}{c}\text { Puls in } \\
10 \text { Secunden }\end{array}$} & \multicolumn{4}{|c|}{ Blutdruck in der A. car.; $\mathrm{mm} \mathrm{Hg}$} \\
\hline & & & Мах. & 1 & Min. & Mittel \\
\hline 24 & 1230 & 29 & 96 & : & 82 & 89 \\
\hline 25 & 1240 & 29 & 94 & & 80 & 87 \\
\hline 26 & 1540 & 30 & 86 & & 78 & 82 \\
\hline 27 & 1550 & 30 & 86 & & 78 & 82 \\
\hline 28 & 1560 & 31 & 87 & & 78 & $82^{1} / 2$ \\
\hline 29 & 1700 & 30 & 92 & 1 & 82 & 87 \\
\hline 30 & 1710 & 30 & 92 & & 82 & 87 \\
\hline 31 & 1720 & 30 & 92 & & 82 & 87 \\
\hline 32 & 1730 & 30 & 92 & j & 82 & 87 \\
\hline 33 & 1740 & 31 & 92 & & 82 & 87 \\
\hline 34 & 1750 & 30 & 92 & & 82 & 87 \\
\hline
\end{tabular}

In der 1760. Sec. wird die Pincette wieder angelegt. In Folge verschiedener Umstände dauert es bis zur 1818. Sec., bevor die $A b$ klemmung ordentlich stattgefunden hat. Die Abklemmung dauert bis zu der 1974. Sec., also 156 Sec. lang. Dabei beträgt der Blutdruck $28^{\mathrm{mm}} \mathrm{Hg}$, und die mittels eines elektrischen Signals angegebene Pulsfrequenz in 10 Sec. resp.: ? $-28-31-29-29-28-$ $24-22-20-21-21-22-19-22-?-?$ In der 1974. Sec. wird die Pincette abgenommen.

\begin{tabular}{|c|c|c|c|c|c|}
\hline \multirow{2}{*}{ Nr. } & \multirow{2}{*}{$\begin{array}{l}\text { Laufende Zeit; } \\
\text { Secunden }\end{array}$} & \multirow{2}{*}{$\begin{array}{c}\text { Puls in } \\
10 \text { Secunden }\end{array}$} & \multicolumn{3}{|c|}{ Blutdruck in der A. car.; $\mathbf{m m ~} \mathrm{Hg}$} \\
\hline & & & Max. & Min. & Mittel \\
\hline 71 & 1974 & 20 & 136 & 28 & - \\
\hline 72 & 1983 & 26 & 148 & 130 & 139 \\
\hline 73 & 1993 & 29 & 156 & 138 & 147 \\
\hline 74 & 2003 & 29 & 156 & 148 & 152 \\
\hline 75 & 2013 & 27 & 156 & 144 & 150 \\
\hline 76 & 2023 & 28 & 152 & 142 & 147 \\
\hline 77 & 2033 & 30 & 150 & 136 & 143 \\
\hline 78 & 2043 & 29 & 144 & 126 & 135 \\
\hline 79 & 2053 & 28 & 134 & 114 & 124 \\
\hline 80 & 2063 & 27 & 122 & 102 & 112 \\
\hline 81 & 2073 & 27 & 110 & 92 & 101 \\
\hline 82 & 2083 & 28 & 99 & 84 & $91^{1 / 2}$ \\
\hline 83 & 2093 & 28 & 91 & 80 & $851 / 2$ \\
\hline 84 & 3003 & 28 & 88 & 76 & 82 \\
\hline 85 & 3013 & 29 & 84 & 76 & 80 \\
\hline 86 & 3023 & 29 & 84 & 75 & $79^{1 / 2}$ \\
\hline
\end{tabular}




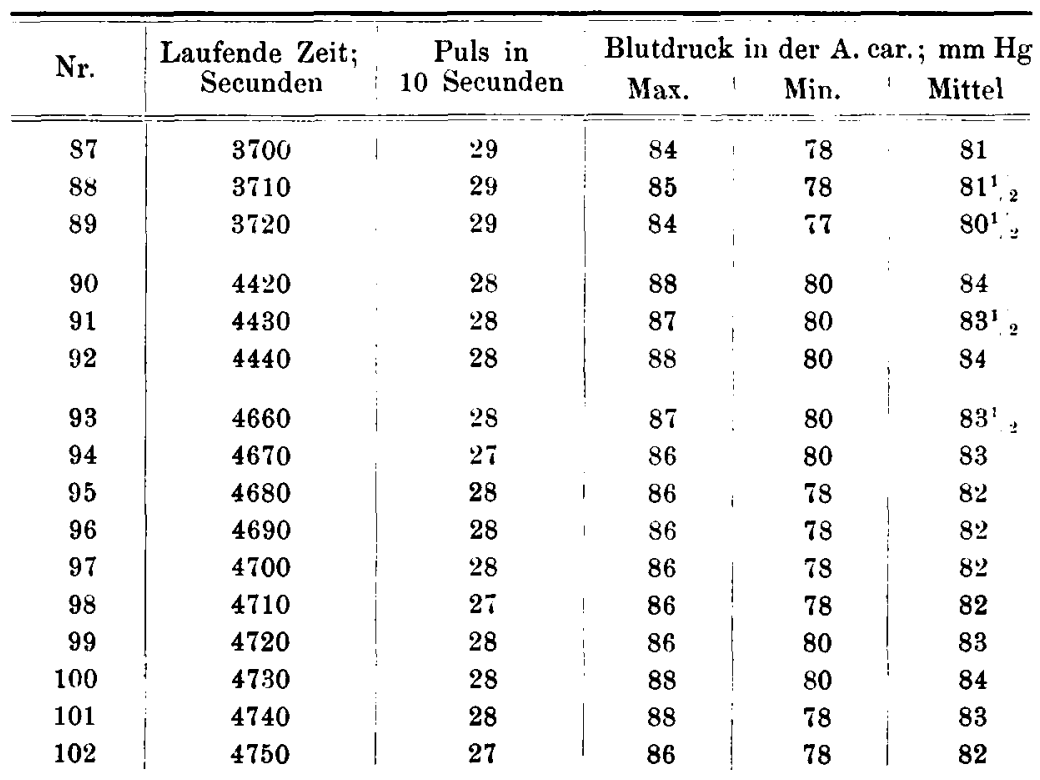

Zwischen der 4760. und 4770. Sec. wird die Pincette angelegt. Die vollständige Abklemmung beginnt um die 4771. Sec. und dauert 192 Sec. lang bis zu der 4963. Sec. Dabei ist der Blutdruck $26{ }^{\mathrm{mn}} \mathrm{Hg}$ und die elektrisch signalisirte Pulsfrequenz in 10 Sec.: ? - ? - 27 $-23-21-23-20-19-13-13-14-14-$ $14-13-14-14-15-10-$ ?

In der 4963. Sec. wird die Pincette weggenommen. Der Blutdruck erhebt sich sogleich, ist aber bis zu der 4993. Sec. nicht messbar, weil das Manometer nicht gut schreibt. Darnach finden wir:

\begin{tabular}{|c|c|c|c|c|c|}
\hline \multirow{2}{*}{ Nr. } & \multirow{2}{*}{$\begin{array}{l}\text { Laufende Zeit; } \\
\text { Secunden }\end{array}$} & \multirow{2}{*}{$\begin{array}{l}\text { Puls in } \\
10 \text { Secunden }\end{array}$} & \multicolumn{3}{|c|}{ Blutdruck in der A. car.; $\mathrm{mm} \mathrm{Hg}$} \\
\hline & & & Max. & Min. & Mittel \\
\hline 103 & 4994 & 24 & 144 & 128 & 136 \\
\hline 104 & 5004 & 25 & 143 & 131 & 137 \\
\hline 105 & 5014 & 25 & 137 & 122 & $129^{1 / 2}$ \\
\hline 106 & 5024 & 26 & 128 & 112 & 120 \\
\hline 107 & 5034 & 26 & 116 & 102 & 109 \\
\hline 108 & 5044 & 26 & 108 & 94 & 101 \\
\hline 109 & 5054 & 25 & 99 & 85 & 92 \\
\hline 110 & 5064 & 24 & 90 & 76 & 83 \\
\hline 111 & 5074 & 24 & 83 & 70 & $76^{1 / 2}$ \\
\hline 112 & 5084 & 24 & 76 & 64 & 70 \\
\hline 113 & 5094 & 24 & 70 & 60 & 65 \\
\hline
\end{tabular}




\begin{tabular}{cccc|c|c|c}
\hline Nr. & $\begin{array}{c}\text { Laufeude Zeit; } \\
\text { Secunden }\end{array}$ & $\begin{array}{c}\text { Puls in } \\
\text { 10 Secunden }\end{array}$ & \multicolumn{3}{c}{ Blutdruck in der A. car.; mm Hg } \\
Max. & Min. & Mittel \\
\hline 114 & 5470 & 27 & 78 & 72 & 75 \\
115 & 5480 & 27 & 78 & 72 & 75 \\
116 & 5490 & 26 & 80 & 74 & 77 \\
117 & 5830 & 27 & 85 & 82 & 85 \\
118 & 5840 & 28 & 88 & 80 & 84 \\
119 & 5850 & 27 & 86 & 80 & 83 \\
120 & 5860 & 28 & & 86 & 82 & 84
\end{tabular}

Im Versuch I ist der mittlere Druck vor der Abklemmung, wahrscheinlich wegen der Narcose, sehr niedrig, 25-33 mm Hg. Während der ersten Abklemmung, welche 115 Sec. lang dauert, sinkt der Druck auf $6-8 \mathrm{~mm}$ herab, erhebt sich aber nach wieder hergestellter Blutcirculation auf $116 \mathrm{~mm} H \mathrm{Hg}$ (Nr. 15) und sinkt dann wieder allmählich herab, so dass er vor der zweiten Abklemmung nur etwa $32 \mathrm{~mm}$ beträgt. Die zweite Abklemmung dauert 180 Sec. lang; 50 Sec. nach dem Beginn derselben ist der Blutdruck auf $5 \mathrm{~mm} \mathrm{Hg}$ herabgesunken. Nach Fortnahme der Pincette schlägt das Herz nur langsam, der mittlere Blutdruck erhebt sich nicht höher als auf $13^{\mathrm{mm}} \mathrm{Hg}$, die Herzschläge sind aber vollkommen normal und zeigen keine Spur eines Deliriums.

Im Versuch II ist rom Anfang an der mittlere Blutdruck etwa $90 \mathrm{~mm}$ Hg. Bei der ersten, 134 Sec. lang dauernden Abklemmung sinkt der Druck auf $30 \mathrm{~mm} \mathrm{Hg}$, und erhebt sich nach Fortnahme der Pincette auf $171 \mathrm{~mm}$ (Per. 10), um darnach wieder auf etwa den ursprünglichen Werth herabzusinken. Eine zweite Abklemmung von 156 Sec. Dauer zeigt ganz dasselbe: Herabsinken des Blutdruckes auf $28 \mathrm{~mm} \mathrm{Hg}$, Steigerung des mittleren Blutdruckes nach beendeter Abklemmung auf $152 \mathrm{~mm} \mathrm{Hg}$ mit darauffolgender Druckabnahme auf $82^{\mathrm{mm}}$. Auch eine dritte Abklemmung von 192 Sec. Dauer giebt ganz dieselben Resultate.

Im Versuch I schlug das Herz nach dem Ende der ersten Abklemmung vollständig regelmässig und normal 24 Mlinuten, bis die zweite Abklemmung stattfand und sogar nach dieser zeigte sich das Herz fortwährend leistungsfähig. Die erste Abklemmung hat also, trotzdem der Blutdruck während derselben auf $6-8 \mathrm{~mm}$ Hg herabgesunken war, an und für sich keine merkbare Beschädigung des Herzens ausgeübt, was vielleicht am Besten daraus hervorgeht, dass der Blutdruck nach dem Ende der Abklemmung einen verhältnissmässig hohen Werth erreicht hat. Diese Blutdrucksteigerung ist aller 
Wahrscheinlichkeit nach ron einer durch die asphyctische Reizung der Gefässcentren hervorgerufenen Gefässcontraction bedingt. Bei einem wenig leistungsfähigen Herzen hätte aber diese Gefässcontraction keine derartige Drucksteigerung hervorrufen können.

Der Versuch II ist in einer gewissen Beziehung nicht gut ausgefallen: Während der Abklemmung sinkt der Blutdruck nicht genügend tief herab. Wenn trotz der um die Vorhöfe gelegten Pincette der Aortadruck $25-30^{\mathrm{mm}} \mathrm{Hg}$ beträgt, so wird natürlich eine Blutströmung durch die Kranzgefässe stattfinden können und dieser Versuch ist also in Bezug auf die uns hier beschäftigende Frage ganz bedeutungslos. Ich habe denselben hier mitgetheilt, um zu zeigen, in einem wie hohen Grade die Reizung der Vasoconstrictoren, welche durch die Aufhebung des Kreislaufes hervorgerufen ist, das Hinüberströmen des Blutes aus den Arterien nach den Venen rerhindern kann und wie in Folge dessen die Arterien, trotzdem sie keine Zufuhr aus dem Herzen erhalten, dennoch von Blut gefüllt bleiben können.

3. Im folgenden Versuch habe ich den Kreislauf nach dem Vorgange von Stefani in der Weise aufgehoben, dass ich mittels einer in das Pericardium eingebundenen Canüle Salzwasser unter einem so hohen Drucke in die Pericardialhöhle eingegossen habe, dass die Blutzufuhr nach dem Herzen aus den Venen unterbrochen worden ist. Das Thier erhielt nur Curare, weil es ja möglich wäre, dass Morphin und Atropin in irgend einer Weise die Resultate der früheren Versuche beeinflusst hätten.

Versuch III. 13. April 1892. Hund 6700 grm. Allein Curare in subcutaner Einspritzung.

\begin{tabular}{c|c|c|c|c|c}
\hline Nr. & $\begin{array}{c}\text { Laufende Zeit; } \\
\text { Secunden }\end{array}$ & $\begin{array}{c}\text { Puls in } \\
\text { 10 Secunden }\end{array}$ & \multicolumn{3}{|c|}{$\begin{array}{c}\text { Blutdruck in der A. car.; mm Hg } \\
\text { Max. }\end{array}$} \\
\hline 1 & 0 & 25 & 176 & 160 & 168 \\
Min. & Mittel \\
2 & 10 & 25 & 178 & 160 & 169 \\
3 & 20 & 30 & 173 & 154 & $1631 / 2$ \\
4 & 30 & $?$ & 166 & 132 & 149 \\
5 & 40 & 31 & 156 & 130 & 143 \\
6 & 50 & 31 & 156 & 146 & 151
\end{tabular}

In der 60. Sec. wird die Blutzufuhr zu dem Herzen durch Erhöhung des intrapericardialen Druckes allmählich aufgehoben. Der minimale Blutdruck beträgt in der 60.-70. Sec.: 48 , in der 70. bis 80 Sec.: 28, von der 80. bis zum Ende der Absperrung $22 \mathrm{~mm}$ Von der 90. Sec. an zeichnet das Manometer keine Blutdrucks- 
schwankungen mehr. Der intrapericardiale Druck beträgt $23^{\mathrm{cm}}$ Wasser. Die Absperrung dauert 146 Sec. lang und wird an der 236. Sec. aufgehoben.

Zwischen der 236. und 359. Sec. schreibt das Manometer schlecht, so dass die Pulsfrequenz nicht gezählt werden kann. An der 259 Sec. ist der Blutdruck auf $198 \mathrm{~mm} \mathrm{Hg}$ gestiegen. Von der 359. Sec. an begegnen wir folgenden Werthen:

\begin{tabular}{|c|c|c|c|c|c|}
\hline \multirow{2}{*}{ Nr. } & \multirow{2}{*}{$\begin{array}{l}\text { Laufende Zeit; } \\
\text { Secunden }\end{array}$} & \multirow{2}{*}{$\begin{array}{l}\text { Puls in } \\
10 \text { Secunden }\end{array}$} & \multicolumn{3}{|c|}{ Blutdruck in der A. car.; $\mathrm{mm} \mathrm{Hg}$} \\
\hline & & & Max. & Min. & Mittel \\
\hline 7 & 359 & 11 & 158 & 108 & 133 \\
\hline 8 & 369 & 15 & 144 & 110 & 127 \\
\hline 9 & 379 & 18 & 140 & 112 & 126 \\
\hline 10 & 389 & 21 & 136 & 122 & 129 \\
\hline 11 & 399 & 23 & 130 & 117 & $1231 / 2$ \\
\hline 12 & 409 & 27 & 126 & 114 & 120 \\
\hline 13 & 419 & 28 & 122 & 112 & 117 \\
\hline 14 & 429 & 27 & 122 & 112 & 117 \\
\hline 15 & 439 & 27 & 122 & 110 & 116 \\
\hline 16 & 449 & 28 & 122 & 108 & 115 \\
\hline 17 & 459 & 28 & 122 & 113 & 117 \\
\hline 18 & 810 & 30 & 108 & 100 & 104 \\
\hline
\end{tabular}

Es wird noch zweimal die Blutzufuhr zu dem Herzen in derselben Weise und mit demselben Ergebniss abgesperrt. Das Herz fâhrt dessenungeachtet fort ganz normal zu schlagen.

Am Ende des Versuches finden wir

\begin{tabular}{c|c|c|c|c|c}
\hline \hline Nr. & $\begin{array}{c}\text { Laufende Zeit; } \\
\text { Secunden }\end{array}$ & $\begin{array}{c}\text { Puls in } \\
\text { 10 Secunden }\end{array}$ & \multicolumn{3}{|c|}{ Blutdruck in der A. car.; mm Hg } \\
Max. & Min. & Mittel \\
\hline 18 & 3030 & 30 & 100 & 94 & 97 \\
19 & 3390 & 30 & 108 & 100 & 104 \\
20 & 3810 & 30 & 110 & 104 & 107
\end{tabular}

Die Compression des Herzens durch Erhöhung des intrapericardialen Druckes hat 146 Sec. lang gedauert; schon nach 30 Sec. zeigten sich am Manometer keine pulsatorischen Druckschwankungen mehr: die Blutzufuhr zum Herzen war also zu dieser Zeit aufgehoben. Dennoch sinkt der Blutdruck nur auf etwa 22 mm $\mathrm{Hg}$ herab - was ganz wie 
im Versuch II von einer durch den Erstickungsreiz bewirkten Gefässcontraction bedingt sein muss.

Nach Ende der Absperrung steigt der Blutdruck sofort an, und zu gleicher Zeit kommt eine ausgesprochene Vagusreizung zum Vorschein - was bezeugt, dass auch das Vaguscentrum durch das Aufhelsen des Kreislaufes gereizt worden ist.

Fs wurde noch zweimal die Blutzufuhr zu dem Herzen mit demselben Resultate aufgehoben. Trotz derselben schlägt jedoch das Herz noch ganz normal mehr als 1 Stunde nach der ersten Abklemmung.

4. Auch in diesem Versuche sank der mittlere Blutdruck nicht so tief herab, dass aus demselben eindeutige Schlüsse in Bezug auf die Bedeutung der Blutzufuhr gezogen werden könnten.

Um bestimmte Resultate zu erhalten, ging ich bei den folgenden Versuchen in einer anderen Weise zu Wege, und zwar schnitt ich gleich nach Anlegung der Pincette die eine Carotis durch; als nun das Blut frei ausströmen konnte, sank der Blutdruck auf einen sehr niedrigen Werth oder auf Null herab, und die Kranzarterien konnten also keine Blutzufuhr mehr erhalten. Die Thiere waren nur mit Curare bewegungslos gemacht.

Versuch IV. 12. September 1893. Hund, $7500 \mathrm{grm}$. Nur Curare in subcutaner Einspritzung. Manometer in der linken Carotis; die rechte Carotis abgebunden.

\begin{tabular}{|c|c|c|c|c|c|}
\hline \multirow{2}{*}{ Nr. } & \multirow{2}{*}{$\begin{array}{l}\text { Laufende Zeit; } \\
\text { Secunden }\end{array}$} & \multirow{2}{*}{$\begin{array}{c}\text { Puls in } \\
10 \text { Secunden }\end{array}$} & \multicolumn{3}{|c|}{ Blutdruck in der A. car.; mm $\mathrm{Hg}$} \\
\hline & & & Max. & Min. & Mittel \\
\hline 1 & $\mathbf{0}$ & 24 & 102 & 82 & 92 \\
\hline 2 & 10 & 25 & 106 & 92 & 99 \\
\hline
\end{tabular}

Jetzt werden die Vorhöfe durch eine fest zugeschraubte Pincette abgeklemmt und gleich nachher die rechte Carotis durchschnitten. Die Abklemmung und Blutung dauern bis zu der 137 Sec., also 117 Sec. lang. Nach 15 Sec. beträgt der Blutdruck $20 \mathrm{~mm}$, nach 30 Sec. $16 \mathrm{~mm}$ und am Ende der Abklemmung $6 \mathrm{~mm}$. Die Pincette wird in der 137 Sec. abgenommen und die rechte Carotis abgebunden. S. Tabelle S. 11.

In der 1570. Sec. werden die Vorhöfe abgeklemmt und die rechte Carotis gelüftet. Die Abklemmung und Blutentziehung dauern 146 Sec., also bis zur 1716. Sec. 15 Sec. nach der Abklemmung beträgt der Druck $14 \mathrm{~mm}$, nach noch $15 \mathrm{Sec}$. ist er auf $12 \mathrm{~mm}$ herabgesunken. Dann finden wir an der 45. Sec.: $11 \mathrm{~mm}$, an der 60. Sec.: 11, an der 75. Sec.: $10^{1 / 2}$, 


\begin{tabular}{|c|c|c|c|c|c|c|}
\hline \multirow{2}{*}{ Nr. } & \multirow{2}{*}{\multicolumn{2}{|c|}{$\begin{array}{c}\text { Laufende Zeit } \\
\text { Secunden }\end{array}$}} & \multirow{2}{*}{$\begin{array}{l}\text { Puls in } \\
10 \text { Secunden }\end{array}$} & \multicolumn{3}{|c|}{ Blutdruck in der A. car.; $\mathrm{mm} \mathrm{Hg}$} \\
\hline & & & & Max. & Min. & Mittel \\
\hline 8 & & 137 & 11 & 80 & - & - \\
\hline 9 & & 142 & 26 & 182 & 144 & 163 \\
\hline 10 & & 152 & 21 & 178 & 134 & 156 \\
\hline 11 & & 162 & 20 & 170 & 138 & 154 \\
\hline 12 & & 172 & 16 & 174 & 116 & 145 \\
\hline 13 & & 182 & 13 & 170 & - & - \\
\hline 14 & & 192 & 13 & 168 & - & - \\
\hline 15 & & 202 & 14 & 160 & 104 & 132 \\
\hline 16 & & 212 & 17 & 144 & 106 & 125 \\
\hline 17 & & 222 & $1 \tau$ & 140 & 106 & 123 \\
\hline 18 & & 232 & 19 & 126 & 102 & 114 \\
\hline 19 & & 242 & 20 & 118 & 98 & 108 \\
\hline 20 & & 252 & 20 & 112 & 92 & 102 \\
\hline 21 & & 262 & 21 & 110 & 90 & 100 \\
\hline 22 & & 272 & 22 & 110 & 94 & 102 \\
\hline 23 & & 282 & 22 & 110 & 94 & 102 \\
\hline 24 & & 292 & 24 & 110 & 96 & 103 \\
\hline 25 & & 302 & 25 & 114 & 100 & 107 \\
\hline 26 & & 312 & 25 & 114 & 102 & 108 \\
\hline 27 & & 322 & 26 & 112 & 102 & 107 \\
\hline 28 & & 332 & 26 & 112 & 102 & 107 \\
\hline 29 & & 342 & 27 & 112 & 102 & 107 \\
\hline 30 & & 352 & 27 & 112 & 102 & 107 \\
\hline 31 & & 362 & 28 & 116 & 108 & 112 \\
\hline 32 & & 372 & 28 & 116 & 106 & 111 \\
\hline 33 & & 382 & 28 & 114 & 106 & 110 \\
\hline 34 & & 392 & 28 & 114 & 104 & 109 \\
\hline 35 & & 402 & 29 & 112 & 104 & 108 \\
\hline 36 & & 412 & 29 & 112 & 102 & 107 \\
\hline 37 & & 422 & 29 & 108 & 102 & 105 \\
\hline 38 & & 432 & 29 & 108 & 102 & 105 \\
\hline 39 & & 850 & 31 & 84 & 76 & $\mathbf{8 0}$ \\
\hline 40 & $!$ & 1150 & 32 & 82 & 74 & 78 \\
\hline 41 & & 1450 & 32 & 86 & 70 & 78 \\
\hline 42 & & 1570 & 31 & 86 & 76 & 81 \\
\hline
\end{tabular}

an der 90. Sec.: 9, an der 105. Sec.: 8, an der 120. Sec.: 7 und am Ende der Abklemmung $6{ }^{\mathrm{mm}} \mathrm{Hg}$. Die Pincette wird an der 1716. Sec. abgenommen und die rechte Carotis abgebunden. 


\begin{tabular}{|c|c|c|c|c|c|}
\hline \multirow{2}{*}{$\mathrm{Nr}$. } & \multirow{2}{*}{$\begin{array}{l}\text { Laufende Zeit; } \\
\text { Secunden }\end{array}$} & \multirow{2}{*}{$\begin{array}{l}\text { Puls in } \\
10 \text { Secunden }\end{array}$} & \multicolumn{3}{|c|}{ Blutdruck in der A. car.; mm $\mathrm{Hg}$} \\
\hline & & & Max. & Min. & Mittel \\
\hline 43 & 1716 & 12 & 178 & $\quad-$ & - \\
\hline 44 & 1726 & 24 & 192 & 162 & 177 \\
\hline 45 & 1736 & 22 & 174 & 116 & 145 \\
\hline 46 & 1746 & 17 & 164 & 132 & 148 \\
\hline 47 & 1756 & 19 & 152 & 126 & 139 \\
\hline 48 & 1766 & 15 & 156 & 94 & 125 \\
\hline 49 & 1776 & 12 & 138 & - & - \\
\hline 50 & 1786 & 12 & 100 & - & - \\
\hline 51 & 1796 & 13 & 102 & - & - \\
\hline 52 & 1806 & 17 & 104 & 80 & 92 \\
\hline$\tilde{5} 3$ & 1816 & 21 & 96 & 78 & 87 \\
\hline 54 & 1826 & 22 & 88 & 74 & 81 \\
\hline $5 \overline{\mathbf{j}}$ & 1836 & 23 & 84 & 70 & 77 \\
\hline 56 & 1846 & 23 & 82 & 70 & 76 \\
\hline 57 & 1856 & 24 & 80 & 70 & 75 \\
\hline 58 & 1866 & 25 & 78 & 68 & 73 \\
\hline 59 & 1876 & 26 & 80 & 68 & 74 \\
\hline 60 & 1886 & 26 & 82 & 72 & 77 \\
\hline 61 & 1896 & 26 & 82 & 74 & 78 \\
\hline 62 & 1906 & 27 & 84 & 74 & 79 \\
\hline 63 & 1916 & 26 & 88 & 80 & 84 \\
\hline 64 & 1926 & 27 & 88 & 80 & 84 \\
\hline 65 & 1936 & 27 & 86 & 76 & 81 \\
\hline 66 & 1946 & 27 & 84 & 76 & 80 \\
\hline 67 & 1956 & 27 & 84 & 76 & 80 \\
\hline 68 & 1966 & 28 & 88 & 78 & 83 \\
\hline 69 & 1976 & 28 & 88 & 80 & 84 \\
\hline 70 & 1986 & 28 & 92 & 80 & 86 \\
\hline 71 & 2350 & 31 & 76 & 68 & 72 \\
\hline 72 & 2360 & 30 & 86 & 74 & 80 \\
\hline 73 & 2370 & 30 & 90 & 76 & 83 \\
\hline
\end{tabular}

Während der 72. Periode fängt eine Erstickung an, welche 60 Sec. lang dauert. Dabei erreicht der Blutdruck den maximalen Werth von $260 \mathrm{~mm}$ Hg. Nach dem Ende der Erstickung fanden wir

\begin{tabular}{c|c|c|c|c|c}
\hline Nr. & $\begin{array}{c}\text { Laufende Zeit; } \\
\text { Secunden }\end{array}$ & $\begin{array}{c}\text { Puls in } \\
\text { 10 Secunden }\end{array}$ & \multicolumn{3}{|c|}{ Blutdruck in der A. car.; mm Hg } \\
Max. & Min. & Mittel \\
\hline 74 & 2440 & 30 & 176 & 134 & 155 \\
75 & 2450 & 30 & 144 & 120 & 132 \\
76 & 2460 & 29 & 132 & 112 & 122 \\
77 & 2470 & 30 & 120 & 108 & 114
\end{tabular}




\begin{tabular}{cccc|c|c|c}
\hline \hline Nr. & $\begin{array}{c}\text { Laufende Zeit; } \\
\text { Secunden }\end{array}$ & \multicolumn{2}{c}{$\begin{array}{c}\text { Puls in } \\
\text { 10 Secunden }\end{array}$} & \multicolumn{3}{c}{ Blutdruck in der A. car. mm Hg } \\
& Max. & Min. & Mittel \\
\hline \hline 78 & 2480 & 30 & 116 & 104 & 110 \\
79 & 2490 & 30 & 112 & 102 & 107 \\
80 & 2500 & 30 & 108 & 100 & 104 \\
81 & 2510 & 30 & 108 & 100 & 104 \\
82 & 2600 & 30 & 94 & 90 & 92 \\
83 & 2610 & 30 & 94 & 88 & 91 \\
84 & 2620 & 30 & 94 & 88 & 91 \\
85 & 2630 & 30 & 92 & 88 & 90 \\
86 & 2930 & 30 & 76 & 68 & 72 \\
87 & 2940 & 31 & 78 & 68 & 73 \\
88 & 2950 & 30 & 80 & 72 & 76
\end{tabular}

Jetzt wird in die Pericardialhöhle $0.04 \mathrm{grm}^{\mathrm{rm}}$ Cocain in $1{ }^{\mathrm{ccm}}$ physiologischer Kochsalzlösung eingegossen. Der Blutdruck beginnt sofort anzusteigen und erreicht einen sehr hohen Werth. Nach 540 Sec. finden wir:

\begin{tabular}{c|c|c|c|c|c}
\hline \hline \multirow{2}{*}{ Nr. } & $\begin{array}{c}\text { Laufende Zeit; } \\
\text { Secunden }\end{array}$ & \multicolumn{2}{|c|}{$\begin{array}{c}\text { Puls in } \\
\text { 10 Secunden }\end{array}$} & \multicolumn{3}{|c|}{ Blutdruck in der A. car.; mm Hg } \\
& Max. & Min. & Mittel \\
\hline 89 & 3490 & 28 & 188 & 176 & 182 \\
90 & 3500 & 28 & 186 & 174 & 180 \\
91 & 3510 & 28 & 182 & 170 & 176
\end{tabular}

An der 4270 Sec. nach dem Beginn des Versuches wird wieder dieselbe Menge von Cocainlösung in die Pericardialhöhle eingegossen. Der Druck erhebt sich noch weiter und wir finden in der 4500. Secunde die folgenden Werthe:

\begin{tabular}{c|c|c|c|c|c}
\hline \hline Nr. & $\begin{array}{c}\text { Laufende Zeit; } \\
\text { Secunden }\end{array}$ & $\begin{array}{c}\text { Puls in } \\
\text { 10 Secunden }\end{array}$ & \multicolumn{3}{|c|}{ Blutdruck in der A. car.; mm Hg } \\
Max. & Min. & Mittel \\
\hline \hline 92 & 4500 & 24 & 202 & 192 & 197 \\
93 & 4510 & 25 & 200 & 188 & 194 \\
94 & 4520 & 24 & 196 & 180 & 188 \\
95 & 4530 & 24 & 190 & 180 & 185 \\
96 & 4540 & 24 & 186 & 176 & 181
\end{tabular}

Dann werden die Vorhöfe nochmals abgeklemmt und die rechte Carotis gelüftet. Die Abklemmung und Blutung dauern 128 Sec., bis zur 4668. Sec. Der Blutdruck beträgt nach 15 Sec.: 16, nach 30 Sec.: 14, nach 45 Sec.: 12, nach 60 Sec.: 11 , nach 75 Sec.: 10 , nach 90 Sec. 
10, nach 105 Sec.: 10, nach 120 Sec.: 10, und am Ende der Abklemmung $10^{\mathrm{mm}} \mathrm{Hg}$. Darnach finden wir

\begin{tabular}{r|cc|c|c|c}
\hline Nr. & $\begin{array}{c}\text { Laufende Zeit; } \\
\text { Secunden }\end{array}$ & $\begin{array}{c}\text { Puls in } \\
\text { 10 Secunden }\end{array}$ & \multicolumn{3}{|c}{ Blutdruck in der A. car.; mm } \\
Max. & Min. & Mittel \\
\hline 97 & 4668 & 11 & 62 & - & - \\
98 & 4713 & 12 & 82 & - & - \\
99 & 4723 & 15 & 94 & - & - \\
100 & 4733 & 17 & 126 & - & - \\
101 & 4743 & 21 & 132 & 116 & 124 \\
102 & 4753 & 23 & 136 & 124 & 130 \\
103 & 4763 & 24 & 140 & 128 & 134 \\
104 & 4773 & 24 & 142 & 132 & 137 \\
105 & 4783 & 24 & 140 & 130 & 135 \\
106 & 4793 & 24 & 138 & 128 & 133 \\
107 & 4803 & 25 & 136 & 122 & 129 \\
108 & 4813 & 25 & 132 & 118 & 125 \\
109 & 4823 & 24 & 126 & 114 & 120 \\
110 & 4833 & 25 & 122 & 110 & 116
\end{tabular}

Das Thier erhält jetzt Atropin in die Pericardialhöhle, was jedoch keine Einwirkung ausübt. In der 5130. Sec. wird das Thier erstickt. Die Pulsfrequenz hält sich dabei um 21-22 pro Sec. und der Blutdruck erhebt sich auf den Werth von $182 \mathrm{~mm}$ Hg. 135 Sec. nach dem Beginn der Erstickung finden wir folgende Werthe:

\begin{tabular}{c|c|c|c|c|c}
\hline \hline \multirow{2}{*}{ Nr. } & $\begin{array}{c}\text { Laufende Zeit; } \\
\text { Secunden }\end{array}$ & \multicolumn{2}{|c|}{$\begin{array}{c}\text { Puls in } \\
\text { Secunden }\end{array}$} & \multicolumn{3}{|c|}{$\begin{array}{c}\text { Blutdruck in der A. car.; mm Hg } \\
\text { Max. }\end{array}$} & Min. & Mittel \\
\hline 111 & 5265 & 24 & 162 & 138 & 150 \\
112 & 5275 & 24 & 144 & 114 & 129
\end{tabular}

Der Versuch wird jetzt unterbrochen.

Versuch V. 17. November 1893 . Hund, $5000 \mathrm{grm}$. Nur Curare in subcutaner Einspritzung. Manometer in der linken Corotis; die rechte Carotis abgebunden.

\begin{tabular}{c|c|c|c|c|c}
\hline \hline Nr. & $\begin{array}{c}\text { Laufende Zeit; } \\
\text { Secunden }\end{array}$ & $\begin{array}{c}\text { Puls in } \\
\text { 10 Secunden }\end{array}$ & \multicolumn{3}{|c|}{ Blutdruck in der A. car.; mm Hg } \\
& Max. & Min. & Mittel \\
\hline 1 & 0 & 34 & 158 & 142 & 150 \\
2 & 10 & 34 & 149 & 139 & 144
\end{tabular}

Jetzt werden die Vorhöfe durch eine fest zugeschraubte Pincette abgeklemmt und unmittelbar nachher die rechte Carotis durch- 
schnitten. Die Abklemmung und Blutung dauern bis zu der 170 Sec., also 150 Sec. lang.

Nach 25 Sec. beträgt der Blutdruck $2 \mathrm{~mm} \mathrm{Hg}$, nach 70 Sec. $1 \mathrm{~mm} \mathrm{Hg}$, nach etwa $85 \mathrm{Sec}$. ist er auf $0 \mathrm{~mm} \mathrm{Hg}$ herabgesunken.

Die Pincette wird in der 170. Sec. abgenommen und die rechte Carotis abgebunden. Der Blutdruck steigt sofort an und erreicht innerhalb 14 Sec. den Werth von $184 \mathrm{~mm}$ Hg. Darnach finden wir

\begin{tabular}{c|c|c|c|c|c}
\hline Nr. & $\begin{array}{c}\text { Laufende Zeit; } \\
\text { Secunden }\end{array}$ & $\begin{array}{c}\text { Puls in } \\
\text { Pecunden }\end{array}$ & \multicolumn{3}{|c}{ Blutdruck in der A. car.; mm Hg } \\
3 & 10 Sec. & Min. & Mittel \\
3 & 185 & 36 & 194 & 166 & 175
\end{tabular}

Nun zeigt sich zuerst eine 50 Sec. lang dauernde Retardation der Herzschläge (Vagusreizung), nach welcher das Herz arhytmisch schlägt, indem die Herzschläge mit einer gewissen Periodicität umwechselnd frequent und langsam sind. Einige Minuten später ist die Herzthätigkeit wieder ganz regelmässig und wir erhalten

\begin{tabular}{cc|c|c|c|c}
\hline Nr. & $\begin{array}{c}\text { Laufende Zeit; } \\
\text { Secunden }\end{array}$ & Puls in & \multicolumn{3}{c}{ Blutdruck in der A. car.; mm Hg } \\
& 10 Secunden & Max. & Min. & Mittel \\
4 & 450 & 31 & 114 & 106 & 110 \\
5 & 460 & 30 & 110 & 100 & 105 \\
6 & 470 & 30 & 102 & 94 & 98 \\
7 & 480 & 31 & 96 & 90 & 93 \\
8 & 490 & 30 & 92 & 87 & $89^{1 / 2}$
\end{tabular}

In der 550. Sec. wird die künstliche Athmung unterbrochen. Der Druck steigt an und erreicht an der 595. Sec. den maximalen Werth von $234 \mathrm{~mm} \mathrm{Hg}$.

Bei der in der 600. Sec. wieder eingeleiteten Athmung stellen sich folgende Werthe dar:

\begin{tabular}{|c|c|c|c|c|c|}
\hline \multirow{2}{*}{ Nr. } & \multirow{2}{*}{$\begin{array}{l}\text { Laufende Zeit; } \\
\text { Secunden }\end{array}$} & \multirow{2}{*}{$\begin{array}{c}\text { Puls in } \\
10 \text { Secunden }\end{array}$} & \multicolumn{3}{|c|}{ Blutdruck in der A. car.; mm $\mathrm{Hg}$} \\
\hline & & & Max. & Min. & Mittel \\
\hline 9 & 600 & 20 & 216 & 168 & 192 \\
\hline 10 & 610 & 29 & 174 & 144 & 159 \\
\hline 11 & 620 & 32 & 147 & 126 & $136^{1} / 2$ \\
\hline 13 & 660 & 34 & 118 & 112 & 115 \\
\hline 14 & 670 & 33 & 116 & 110 & 113 \\
\hline 15 & 920 & 33 & $\mathbf{7 5}$ & 71 & 73 \\
\hline 16 & 930 & 33 & 75 & 70 & $721 \%$ \\
\hline
\end{tabular}


Dann wird das 'Thier während 130 Sec. erstickt, wobei die Verhältnisse sich ganz wie bei der früheren Erstickung verhalten. 270 Sec. nach dem Ende dieser Erstickung finden wir:

\begin{tabular}{c|c|c|c|c|c}
\hline Nr. & $\begin{array}{c}\text { Laufende Zeit; } \\
\text { Secunden }\end{array}$ & $\begin{array}{c}\text { Puls in } \\
\text { 10 Secunden }\end{array}$ & \multicolumn{3}{|c|}{ Blutdruck in der A. car.; mm Hg } \\
Max. & Min. & Mittel \\
\hline 17 & 1330 & 30 & 126 & 122 & 124 \\
18 & 1340 & 31 & 123 & 120 & $121^{1 / 2}$
\end{tabular}

Die Pincette wird nun zum zweiten Mal angelegt und die rechte Carotis durchschnitten. Die Abklemmung dauert 200 Sec. Nach Lösung derselben stellt sich sofort Herzdelirium ein und das Thier stirbt.

Im Versuche IV dauert die erste Abklemmung und Blutung 117 Sec. Dabei ist der Blutdruck nach 30 Sec. auf $16 \mathrm{~mm}$ und am Ende der Abklemmung auf $6 \mathrm{~mm} \mathrm{Hg}$ herabgesunken. Nach Ende derselben erreicht der Blutdruck einen sehr hohen Werth und sinkt dann wieder herab.

Die zweite Abklemmung und Blutung dauert $146 \mathrm{Sec}$., wobei der Druck nach 30 Sec. auf $12 \mathrm{~mm}$, nach 45 Sec. auf 11 , nach 90 Sec. auf 9 und am Ende der Abklemmung auf $6 \mathrm{~mm}$ Hg herabgesunken ist. Bei wiederhergestelltem Kreislauf stellen sich die Verhältnisse ganz wie nach der ersten Abklemmung dar.

Eine 60 Sec. lang dauernde Erstickung treibt den Blutdruck auf den hohen Werth von $260 \mathrm{~mm}$ und in der 2950 Sec. (2800 Sec. nach dem Ende der ersten Abklemmung und Blutung) ist der mittlere Druck noch $76^{\mathrm{mm}}$ hoch. Die beiden Abklemmungen haben also trotz des dabei stattfindenden niedrigen Druckes - die Leistungsfähigkeit des Herzens gar nicht herabgesetzt.

In der 2960. Sec. wird in der Pericardialhöhle 0.04 grm Cocain in $1 \mathrm{~cm}$ Salzwasser eingegossen, in Folge dessen der Blutdruck erheblich ansteigt. Bei jetzt in der 4550. Sec. vom Beginn des Versuches an gerechnet stattfindender, 128 Sec. lang dauernder, Abklemmung und Blutung finden wir den Blutdruck nach 30 Sec. auf 14, nach 75 Sec. auf $10 \mathrm{~mm} H g$ herabgesunken. Nachdem der Kreislauf wieder hergestellt worden ist, steigt der Drack wieder auf einen ziemlich hohen Werth an. 5138 Sec. nach dem Ende der ersten Abklemmung und Blutung wird der Versuch unterbrochen, der mittlere Blutdruck ist dabei noch $129 \mathrm{~min} \mathrm{Hg}$.

Im Versuche $\mathrm{V}$ sinkt der mittlere Blutdruck bei der ersten $\mathrm{Ab}$ klemmung und Blutung innerhalb 25 Sec. auf $2 \mathrm{~mm} \mathrm{Hg}$ und später, nach etwa 60 Sec., auf $0 \mathrm{~mm} H \mathrm{Hg}$ herab. Die Abklemmung und 
Blutung dauern 150 Sec. Gleich nach dem Ende derselben erhebt sich der Blutdruck wieder und erreicht innerhalb 14 Sec. den Werth von $184^{\mathrm{mm}} \mathrm{Hg}$. 1180 Sec. nach dem Ende der Abklemmung schlägt das Herz rollkommen normal; der mittlere Blutdruck ist dabei noch etwa $122 \mathrm{~mm} \mathrm{Hg}$.

5. Ich stelle die Versuche übersichtlich zusammen:

\begin{tabular}{|c|c|c|c|c|c|c|}
\hline 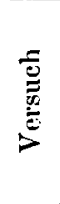 & $\begin{array}{l}\text { Mittlerer } \\
\text { Blutdruck vor } \\
\text { der ersten } \\
\text { Abklem- } \\
\text { mung; } \\
\text { mm Hg }\end{array}$ & $\begin{array}{c}\text { Dauer der } \\
\text { ersten } \\
\text { Abklem- } \\
\text { mung; } \\
\text { Secunden }\end{array}$ & $\begin{array}{c}\text { Druckmin. } \\
\text { während } \\
\text { der ersten } \\
\text { Abklem- } \\
\text { mung; } \\
\text { mm Hg }\end{array}$ & $\begin{array}{c}\text { Druck- } \\
\text { max. nach } \\
\text { der ersten } \\
\text { Abklem- } \\
\text { mung: } \\
\text { mm Hg }\end{array}$ & $\begin{array}{c}\text { Das Herz } \\
\text { bleibt nach } \\
\text { der ersten } \\
\text { Abklemmung } \\
\text { leistungsfahig } \\
\text { wenigst. Sec. }\end{array}$ & $\begin{array}{l}\text { Mittlerer } \\
\text { Blutdruck } \\
\text { am Ende } \\
\text { des Ver- } \\
\text { suches; } \\
\text { mm Hg }\end{array}$ \\
\hline I & $33-25$ & 115 & 6 & 121 & 1596 & 32 \\
\hline II & $88-92$ & 134 & 30 & 182 & 5664 & 84 \\
\hline III & $143-169$ & 146 & 22 & 158 & 3584 & 107 \\
\hline IV & $92-99$ & 117 & 6 & 260 & 5138 & 129 \\
\hline $\mathbf{V}$ & $144-150$ & 150 & 0 & 234 & 1180 & 122 \\
\hline
\end{tabular}

In Cohnheim's und v. Schulthess-Rechberg's Versuchen über die Folgen der Kranzarterienverschliessung für das Herz, welche Versuche die Lehre von der Wirkung eines wirklichen Herzgiftes, das sich während des Verschlusses des Kranzarterienastes gebildet hätte, begründeten, hat der steile Abfall des Blutdruckes und der Herztod bei Verschluss der grösseren Kranzarterienäste in 4 Versuchen in den folgenden Zeiträumen stattgefunden:

1. Vers. vom 18. März 1881. Die Ligatur am R. descend. cor. sin. $10^{\mathrm{mm}}$ vom Ursprung aus der Aorta. Der steile Abfall erfolgt 95 Sec. nach der Ligatur.

2. Vers. vom 7. Mărz 1881. Ligatur des R. descend. $18^{\mathrm{mm}}$ vom Ursprung. Herzstillstand nach 125 Sec.

3. Vers. vom 5. Februar 1881. Ligatur des R. desc. und der ihn begleitenden Venen. Herzstillstand nach 90 Sec.

4. Vers. vom 14. Februar 1881. Ligatur des $R$. circumflexus $12 \mathrm{~mm}$ vom Ursprung. Herzstillstand nach 75 Sec.

Die Zeit, innerhalb welcher die Ljgatur eines grösseren Kranzarterienastes zum Herzstillstand führte, schwankt also zwisehen 75 und 125 Sec. oder beträgt „durchschnittlich kaum zwei Minuten" (Cohnheim und v. Schulthess-Rechberg).

Bei meinen Versuchen war der ganze Kreislauf 115-150 Sec. lang vollstä̀ndig anfgehoben und in keinem einzigen Falle trat das Herzdelirium ein, weder während noch nach der Abklemmung, trotz 
dem das Thier mindestens 1180 See. darnach beobachtet wurde. Die Versuche II und III sind zwar, wie schon oben bemerkt ist, von keiner Bedeutung in dieser Hinsicht, die anderen Versuche sind aber um so beweisender.

Man könnte allerdings bemerken, dass der mittlere Blutdruck im Versuch I sehr niedrig ist und dass also, wie schon Cohnheim und v. Schulthess-Rechberg herrorheben, dies hypothetische Herzgift, wegen zu schwacher Herzcontractionen, in einer zu geringen Menge gebildet worden sei. Diese Bemerkung trifft aber für die Versuche IV und V nicht zu: hier ist der Druck vor der Abklemmung 92-99, bezw. 144-150 $\mathrm{mm}$ hoch, und doch ist das Herz, nach einer 117 bezw. 150 Sec. dauernden Abklemmung, vollständig leistungsfähig. Und in diesen Versuchen sinkt während der Abklemmung der mittlere Blutdruck schnell auf einen sehr niedrigen Werth herab.

Ich kann daher nichts anderes finden, als dass diese Versuche, wie übrigens Untersuchungen von mehreren früheren Autoren ganz bestimmt zeigen, dass derjenige Herzstillstand, den Cohn heim und r. SchulthessRechberg beobachtet haben, nicht durch die Anämie eines umschriebenen Theiles der Herzwand, sondern durch Nebenverletzungen bedingt ist. 